\title{
Quality Inspection of Necked-in Area in Pen Point based on Machine Vision
}

\author{
Tang Liping ${ }^{1, ~ a, ~ C h e n ~ F e n ~}{ }^{1, b,{ }^{*}}$, Guo Hengchang ${ }^{2, c}$ \\ ${ }^{1}$ School of Economics and Management, Nanjing University of Science and Technology, Nanjing, \\ Jiangsu Province, China \\ ${ }^{2} \mathrm{M} \& \mathrm{G}$ Chenguang Stationery co., Ltd., Shanghai, China

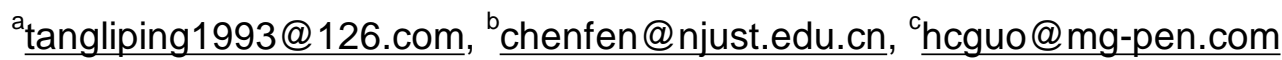 \\ * Corresponding Author: Chen Fen, associate professor, chenfen@ njust.edu.cn
}

\begin{abstract}
Keywords: Halcon; Machine vision; Edge detection; Size detection; Defect detection
Abstract. In order to solve the low efficiency and low accuracy of manual inspection in the quality of necked-in area in pen point, a program of pen point quality inspection was developed based on machine vision in Halcon platform. Firstly, use the median filter to remove the image noise and enhance the contrast of the image; then get the object region of the image through Blob analysis and use edge detection to extract the sub-pixel edges of the object region; finally, use the least square method to fit the edges, and detect the defects by the difference image algorithm. Experimental results show that the program can measure the thickness of the necked-in area quickly and accurately, and identify the defect efficiently.
\end{abstract}

\section{Introduction}

"We do not have the ability to produce mold steel, including the ball on the ball pen." Premier Li Keqiang said in 2016. Indeed, the annual production of ball pens in China is about 40 billion, but some of the core components are mainly depends on imports. The Premier's concern raised the exploration of independent research and development and technological innovation in pen industry, including the quality inspection of the pen point. The necked-in area in pen point is an essential component of pens, its quality directly affects the writing performance of the pen. The efficiency and accuracy of the existing manual inspection is very low. It has been unable to reach the requirements of large-scale, high-speed, and real-time in production. Thus, it is crucial to develop a rapid inspection method in practical production.

The non-contact measurement of machine vision technology can not only avoid the damage to the surface of the product but also improve the detection efficiency and accuracy. It has been widely used in agriculture ${ }^{[1]}$, medicine ${ }^{[2]}$, textile ${ }^{[3]}$ electronic manufacturing ${ }^{[4]}$ and other fields. For stationery and office supplies industry, machine vision technology has not been popularized in China. This paper will propose a quality inspection program of the pen point based on Halcon. The acquired image is input into the computer and processed by the image processing algorithms such as threshold segmentation, sub-pixel edge detection, difference matching, and other methods to achieve fast and accurate measurement of the thickness and the locating of defects. The proposed program is simple and easy to implement. It can meet the demand of online quality inspection for the pen point through experimental analysis and test.

\section{Processes of machine vision detection system}

The online machine vision detection system mainly includes three modules: image acquisition, image processing and analysis, and quality inspection. The whole process is shown in Fig.1. 
The necked-in area in pen point is shown in Fig. 2. The production of pen points must go through many procedures, which may bring several appearance defects such as thickness failure and bruises. Therefore, it is necessary to inspect the quality before putting the pen into next procedure, so as to remove unqualified pen points. The main work of the inspection is to measure the thickness of the necked-in area and mark the location of the defects. The standard value of the thickness is $0.025 \pm 0.005 \mathrm{~mm}$.

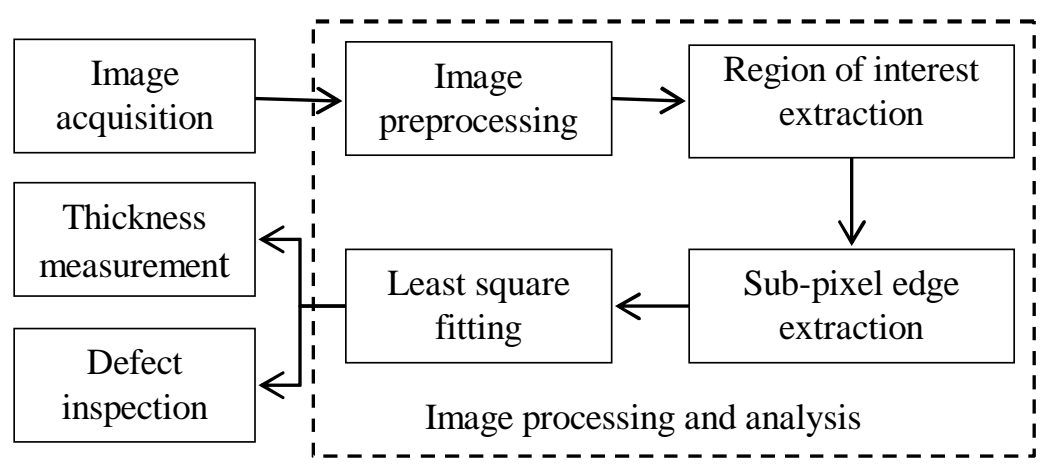

Fig. 1 Machine vision detection processes

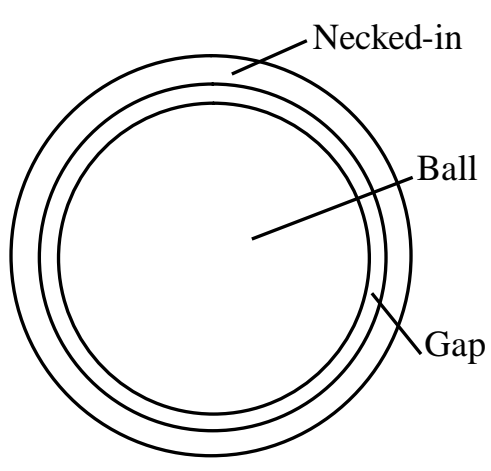

Fig. 2 Structure diagram

\section{Image processing and analysis}

Image preprocessing. The original image acquired is shown in Fig. 3(a). External factors such as uneven illumination, machine vibration, and other factors will inevitably bring interference in the process of image acquisition and transmission, resulting in some random and discrete pixels on the image which is the image noise ${ }^{[5]}$. We use the median filter ${ }^{[6]}$ algorithm to remove the noise in the image. The image after median filter is shown in Fig. 3(b).

After smoothing, the edges and contours of the image are blurred. In order to make the edges more clear and improve the low contrast caused by lack of exposure, image enhancement is necessary. Emphasize() operator in Halcon is used in this paper to enhance the image. The edges of the image are clearer after enhancement as showed in Fig. 3(c).

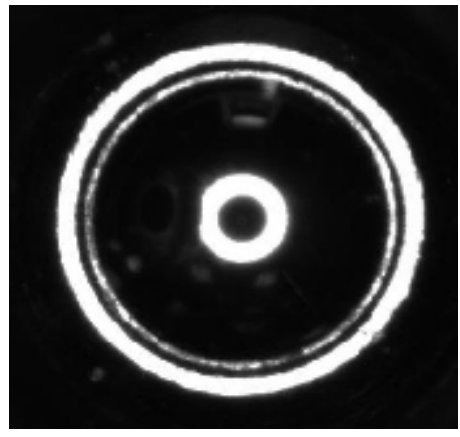

Fig. 3 (a) Original image

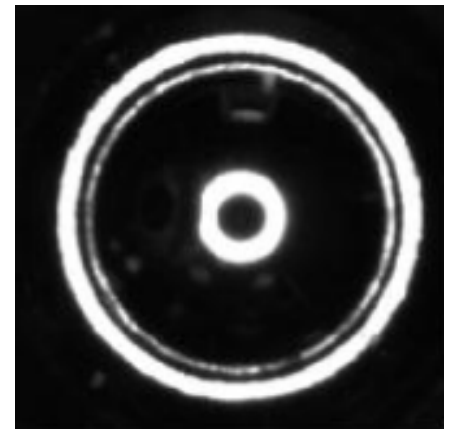

(b) Image filter

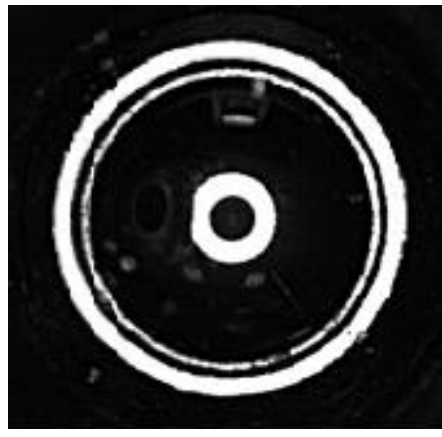

(c) Image enhancement

Region of interest extraction. Image acquisition is operated in bright lighting environment, in which the object is bright, while the background is black. The gray histogram of the image is a bimodal curve (Fig. 4). This simplifies the target segmentation of the necked-in area. Through the operator threshold(), the image is divided into two parts, that is, object and background.

There are multiple objects in the image after threshold segmentation, and may have different degrees of adhesion between the targets. We use the opening_circle() operator to open a region with a radius of 5 circular structuring element to remove interference connected with the necked-in area and suppress small points, and then the closing_circle() operator to close the region with a radius of 15 circular structuring element to fill the broken parts of the necked-in area.

After morphology process, there are several connected regions in the image. In order to make the subsequent image detection contain only the object necked-in area, we use the operator connection() 
to segment the regions from their connectivity, and select the object area through the operator select_shape() according to the prior knowledge. The ROI is shown in Fig. 5.

Sub-pixel edge detection. The edges of the ROI region should be extracted before thickness measurement and defect detection. Edge detection is one of the most common and effective methods for edge extraction and can get relatively accurate edge information of the image ${ }^{[7]}$. But considering the high accuracy requirements of the detection, it is difficult and costly to improve the measurement accuracy by simply increasing the precision of the hardware equipment. Therefore, in this paper we consider the sub-pixel edge detection ${ }^{[8]}$ to extract the edges of the image. Through the operator edges_sub_pix(), the sub-pixel edges of the ROI region are extracted with the canny filter. This operator directly returns the edges in sub-pixel precision as showed in Fig. 6 (red line), and the green line is the edges in pixel level.

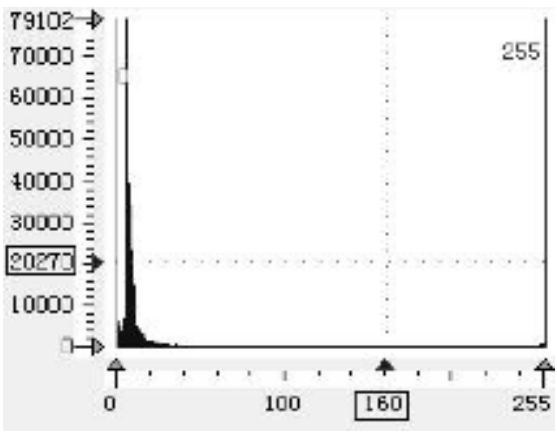

Fig. 4 Gray histogram

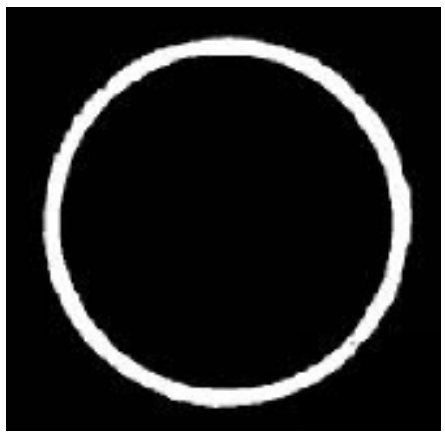

Fig. 5 ROI

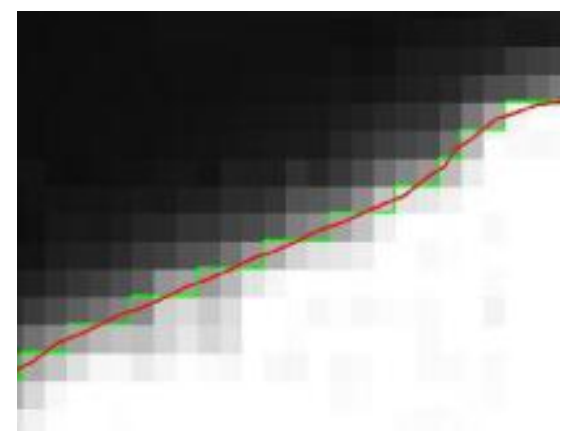

Fig. 6 Sub-pixel edge detection

Least square fitting. Calculating the thickness and detecting the defects of the ROI region need to get the center and radius of the inner and outer circle first. The contours of the ROI are circular, so the least squares method is used to fit the extracted edges. The set of edge points obtained by edge detection is $P(n)$, where $n$ is the number of edge points. The specific process is as follows:

1) Assuming the equation of the circle is $(x-a)^{2}+(y-b)^{2}=r^{2}$, the residual $\varepsilon_{i}$ for any point $\left(x_{i}, y_{\mathrm{i}}\right)$ is:

$\varepsilon_{i}=\left(x_{i}-a\right)^{2}+\left(y_{i}-b\right)^{2}-r^{2}$.

2) The sum of squares of residuals for all edge points $Q(a, b, r)$ is:

$Q(a, b, r)=\sum_{i=1}^{n} \varepsilon_{i}^{2}=\sum_{i=1}^{n}\left[\left(x_{i}-a\right)^{2}+\left(y_{i}-b\right)^{2}-r^{2}\right]^{2}$

3) According to the least squares principle, there is $\frac{\partial Q(a, b, r)}{\partial a}=\frac{\partial Q(a, b, r)}{\partial b}=\frac{\partial Q(a, b, r)}{\partial c}=0$. So the corresponding circle parameters $(a, b, r)$ can be calculated.

In the process of ROI extraction, it can also split out some interference that does not belong to the target object, so there may be some false edges in the extracted edges. We firstly segment the extracted edges by operator segment_contours_xld(), and then use the operator select_shape_xld() to filter edges and remove noise interference. Finally, we union the edges to get the complete edges of the necked-in area, that is the edges to be fitted. The least square circle fitting is used to obtain the centers and radii of the inner and outer circle by operator fit_circle_contours_xld().

\section{Quality detection}

Thickness measurement. Ideally, the inner and outer circle of the necked-in area are concentric circles, whose thickness is the difference between the radii of the two circles. However, due to the inevitable errors in the actual production process, there is a difference between the two centers. So the direct calculation of the difference between the two radii is not accurate enough. This paper calculates the average as the thickness value. We select 5 points from the outer circle to calculate the minimum distance from each point to the inner circle by the operator dist_pc(). The average value of the five results is the final thickness value of the necked-in area. 
Defect inspection. The defects in the necked-in area are mainly bruises and are located in the interior of the outer circle and outside the inner circle (Fig. 7(a)). The gray value between defect area and object area varies greatly. In this paper, we use the difference image method to detect the internal defect of the necked-in area. The difference image method is to calculate the absolute values of gray level difference between the image and the template image in each point ${ }^{[9]}$. The absolute values are the gray value of each point. However, in the actual production process, there are differences between products. So the use of a standard template is inapplicable. In this paper, the annular region enclosed by the fitted inner and outer circles is used as the template image to calculate the gray level difference with the necked-in area in the original image. The specific processes are as follows:

1) Get the regions enclosed by the fitted inner and outer circles. Using the operator difference() in Halcon to get the template image;

2) Use the reduce_domain() operator to get the necked-in area, and do the difference with the template image to get the possible defective areas. The result is shown in Fig. 7(b);

3) Segment the possible defective areas from the background by threshold();

4) Use the operator connection() to obtain the connected areas and set the corresponding standard according to the criterion of detection. Select regions whose feature values are greater than the set value as the final defective area. The defective areas are shown in Fig. 7(c) in red.

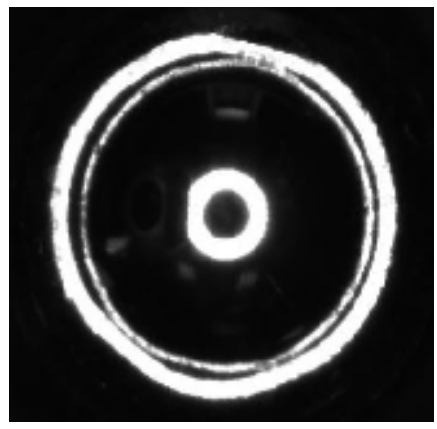

Fig. 7 (a) Bruised image

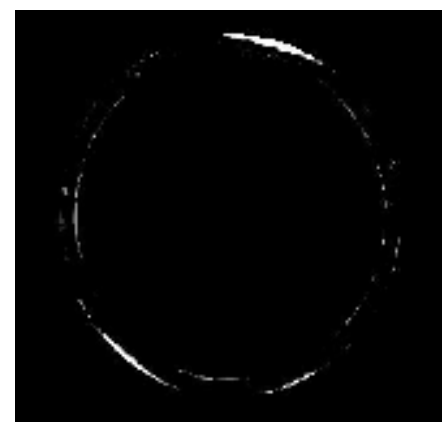

(b) Difference image

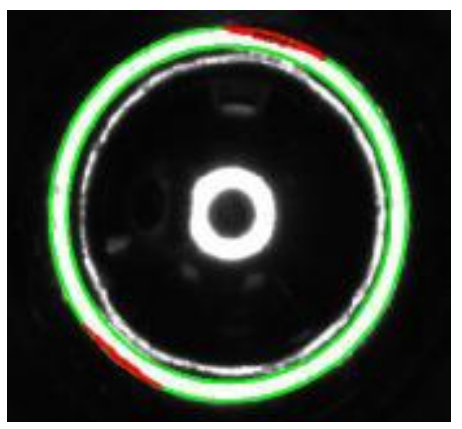

(c) Defective areas

\section{Results analysis}

System calibration. The thickness values are measured in pixel while the real values are in $\mu \mathrm{m}$. To calibrate the system, this article uses the high depth optical microscope VHX-500 to get the real value of the qualified pens' thickness of the necked-in area $(\mu m)$. Calculating the thickness uses the method in this paper (pixel). The conversion factor $\mathrm{K}$ between the actual value and the unit pixel is calculated by $\mathrm{K}=\mathrm{d}_{\mu \mathrm{m}} / \mathrm{d}_{\text {pixel }}$. By several tests, $\mathrm{K}$ value is more accurate to take $1.807 \mu \mathrm{m} / \mathrm{pixel}$.

Experimental results analysis. To test the inspection accuracy, 200 different images of pens are collected for experimentation. Table 1 is 20 testing values in two methods. In terms of measurement accuracy, we use the real value minus the experimentally values to get the relative errors. The absolute values of the relative errors are within $\pm 2 \mu \mathrm{m}$, which can meet the requirement of the enterprise for measurement error.

Table 1 Thickness values in two methods

\begin{tabular}{cccc|cccc}
\hline No. & $\begin{array}{c}\text { Testing } \\
\text { value/ }[\mu \mathrm{m}]\end{array}$ & $\begin{array}{c}\text { Real } \\
\text { value/ }[\mu \mathrm{m}]\end{array}$ & $\begin{array}{c}\text { Relative } \\
\text { error } /[\mu \mathrm{m}]\end{array}$ & No. & $\begin{array}{c}\text { Testing } \\
\text { value/ }[\mu \mathrm{m}]\end{array}$ & $\begin{array}{c}\text { Real } \\
\text { value/ }[\mu \mathrm{m}]\end{array}$ & $\begin{array}{c}\text { Relative } \\
\text { error/ }[\mu \mathrm{m}]\end{array}$ \\
\hline 1 & 25.857 & 26.57 & 0.713 & 11 & 26.097 & 25.13 & -0.967 \\
2 & 26.028 & 26.44 & 0.412 & 12 & 26.203 & 26.87 & 0.667 \\
3 & 27.343 & 26.76 & -0.583 & 13 & 25.895 & 26.38 & 0.485 \\
4 & 26.799 & 26.22 & -0.579 & 14 & 25.519 & 26.32 & 0.801 \\
5 & 25.101 & 25.91 & 0.809 & 15 & 26.788 & 25.44 & -1.348 \\
6 & 26.199 & 26.54 & 0.341 & 16 & 26.985 & 27.46 & 0.475 \\
7 & 25.944 & 26.79 & 0.846 & 17 & 25.922 & 26.43 & 0.508
\end{tabular}




\begin{tabular}{cccc|cccc}
8 & 25.632 & 24.86 & -0.772 & 18 & 24.999 & 25.75 & 0.751 \\
9 & 25.726 & 26.81 & 1.084 & 19 & 26.653 & 27.59 & 0.937 \\
10 & 25.806 & 26.48 & 0.674 & 20 & 24.815 & 25.51 & 0.695 \\
\hline
\end{tabular}

In defect detection, taking the percentage of misjudgment samples in total samples as the false positive rate. As showed in Table 2, the false positive rate is $2 \%$. In the detection speed, the average detection time of a single image is within 200ms. The detection rate is greatly improved compared with the manual inspection, which can meet the actual high-speed production needs of enterprises.

Table 2 Experimental results

\begin{tabular}{cccc}
\hline Samples & $\begin{array}{c}\text { Average measurement } \\
\text { error } /[\mu \mathrm{m}]\end{array}$ & $\begin{array}{c}\text { False positive rate } \\
/[\%]\end{array}$ & $\begin{array}{c}\text { Average time } \\
/[\mathrm{ms}]\end{array}$ \\
\hline 200 & 1.0918 & 2 & 175 \\
\hline
\end{tabular}

\section{Conclusion}

In this paper, a vision quality inspection program in Halcon platform is proposed. The program uses sub-pixel edges detection and difference image method to realize the thickness measurement and defect locating. It can realize a fast, stable, and accurate automatic inspection. Compared with the manual inspection, the vision inspection can reduce the production cost to some extent. The vision inspection proposed in this paper meets the development needs of industrial automation and plays a positive role in promoting the machine vision technology in the office stationary industry.

\section{Acknowledgments}

This paper is supported by the Specific Funds of Shanghai Municipal Commission of Economy and Informatization for Industrial Restructuring and Upgrading "Research on Automatic Inspection Technology and its Application in the Pen Industry based on Machine Vision" (No.: Shanghai CXY-2016-009).

\section{References}

[1] Qureshi W S, Payne A, Walsh K B, et al. Machine vision for counting fruit on mango tree canopies[J]. Precision Agriculture, 2016, 17(3):1-21.

[2] Wu Chengzhong, Wang Yaonan, He Zhendong, et al. Research on foreign insoluble particulate detection method for medicinal solution based on machine vision[J]. Chinese Journal of Scientific Instrument, 2015, 36(7):1451-1461.

[3] Yu Zuyao, Wang Hongyuan, Zhang Ji.Online testing of fabric based on machine vision[J].Computer Engineering and Design, 2016, (10):2851-2856.

[4] Zhou Jun, Xu Shuo, Tu Dawei. Machine vision system based precise positioning for PCB solder Joints[J]. Machine Building \& Automation, 2016, (3):172-175.

[5] Zhen Li. Research on the detection system of rail surface defects based on computer vision[D]. Nanjing University of Aeronautics and Astronautics, 2011.

[6] Yang Z F, Bai J Y. Vial bottle mouth defect detection based on machine vision[C]// IEEE International Conference on Information and Automation. IEEE, 2015:2638-2642.

[7] Jia Yonghong. Digital image process[M]. Wuhan: Wuhan University Press, 2010.

[8] Tang Song, Yang Qihua, LIU Guanghai. Valve core size sub-pix measurement based on Halcon[J]. Computer Engineering and Applications, 2017 ,53(3):237-241. 
[9] Zhao Yufeng, Gao Chao, Wang Jianguo. Research on machine vision based Industrial product surface defect detection algorithm[J]. Computer Applications and Software, 2012, 29(2):152-154. 